\title{
Semi-Markov model of a multi-component energy system with component-wise storages
}

\author{
Yuriy E. Obzherin ${ }^{1}$, Stanislav M. Sidorov ${ }^{1, *}$, and Mikhail M. Nikitin ${ }^{1}$ \\ ${ }^{1}$ Sevastopol State University, Higher mathematics Department, Sevastopol, Russia
}

\begin{abstract}
To increase the reliability and efficiency of the functioning of various purposes systems (in particular, energy systems), time redundancy is used. In this paper, a semi-Markov model of a multicomponent system with component-wise storage devices, which are the sources of the time reserve, is constructed. The stationary characteristics of reliability and efficiency of the system under consideration are found. The analysis of the influence of storage capacities on the reliability and efficiency of the system is been.
\end{abstract}

\section{Introduction}

To ensure the reliability and efficiency of systems, time redundancy is used.

Time redundancy [1-6] provides the system with the ability to use up some additional time (time reserve) to restore performance during operation. For systems with time redundancy, a malfunction of the system is not necessarily accompanied by a system failure, since it is possible to restore the system's operability for a reserve time.

Time redundancy is used in gas transmission systems, in which underground gas storages are the source of time reserve, in electric power systems, time reserve is realized due to energy storage $[5,6]$.

There are problems of modelling systems, and solving optimization problems, taking into account the availability of a reserve of time.

In [6], a semi-Markov model of a two-component system with component-wise storage devices was constructed; stationary characteristics of reliability were found.

In this paper, on the basis of the theory of semiMarkov processes with a common phase state space [710, 12], a semi-Markov model of a multi-component system with a component-wise instantly replenished time reserve is constructed. The stationary characteristics of the reliability and efficiency of the system are found, the influence of the time reserve value on the obtained characteristics is analysed.

\section{Description of the system functioning}

Consider the system $\mathrm{S}$, consisting of $\mathrm{N}$ components, time to failure of which are random variables (RVs) $\alpha_{k}$ with the distribution functions (DFs) $F_{k}(t)$, a restoration times are RVs $\beta_{k}$ with DFs $G_{k}(t), k=\overline{1, N}$.
Each component of the system has a random instantly replenished time reserve [1-3] $\tau_{k}$ with DF $R_{k}(t)$. The time reserve starts to be used at the moment the component recovery starts. A component failure occurs when the component is restored and the time reserve ( $\beta_{k}>\tau_{k}$ ) has been completely used up and continues until the component is restored. By the time the component is restored, the time reserve is replenished to its original value. RVs $\alpha_{k}, \beta_{k}, \tau_{k}$ are assumed to be independent in aggregate, having finite mathematical expectations; DFs $F_{k}(t), G_{k}(t), R_{k}(t)$ have distribution densities $f_{k}(t), g_{k}(t), r_{k}(t)$. The failure of the system $\mathrm{S}$ is determined based on the analysis of the structure of the system. Recovery of system components is assumed unlimited.

\section{Semi-Markov model building}

To describe the functioning of the system $S$, we use the semi-Markov process (SMP) $[8,9] \xi(t)$. We introduce a discrete-continuous phase state space of the form:

$$
\begin{aligned}
& E=\left\{i \bar{i} \bar{x}: \bar{d}=\left(d_{1}, d_{2}, \ldots, d_{k}, \ldots, d_{N}\right),\right. \\
& \left.\bar{x}=\left(x_{1}, x_{2}, \ldots, x_{k}, \ldots, x_{N}\right), x_{k} \geq 0, k=\overline{1, N}\right\},
\end{aligned}
$$

where $i=\overline{1, N}$ is the number of the component in which there was a change in physical condition. Component $d_{k}$ of the vector $\bar{d}$ describes the physical state of the element with the number $k$ :

$$
d_{k}=\left\{\begin{array}{l}
1, \text { if } \mathrm{k}-t h \text { component is operational, } \\
\overline{1}, \text { if } \mathrm{k}-\text { th } \text { component is restored and } \\
\text { functions due to the time reserve, } \\
0, \text { if } \mathrm{k}-t h \text { component is in failure. }
\end{array}\right.
$$

* Corresponding author: xaevec@mail.ru 
The continuous component $x_{k}$ of vector $\bar{x}$ indicates the elapsed time since the last change in the physical state in the component with the number $k$; note that $x_{i}=0$.

Find the residence times in the states of the system. To do this, we introduce the RV:

$$
\delta_{z}^{(k)}=\left\{\begin{array}{l}
\alpha_{k}, \text { if } z=1, \\
\beta_{k} \wedge \tau_{k}, \text { if } z=\overline{1}, \\
{\left[\beta_{k}-\tau_{k}\right]^{+}, \text {if } z=0,}
\end{array}\right.
$$

where $\wedge$ is the minimum sign, $\left[\beta_{k}-\tau_{k}\right]^{+}$is $\mathrm{RV}$, which DF is determined by the equality

$$
P\left(\left[\beta_{k}-\tau_{k}\right]^{+} \leq t\right)=1-\frac{\int_{0}^{\infty} \bar{G}_{k}(y+t) r_{k}(y) d y}{P\left(\beta_{k}>\tau_{k}\right)} .
$$

We denote by $V_{z}^{(k)}(t)=P\left(\delta_{z}^{(k)}<t\right)$ the DFs of RVs $\delta_{z}^{(k)}, \bar{V}_{z}^{(k)}(t)=1-V_{z}^{(k)}(t), v_{z}^{(k)}(t)$ is probability densities of RVs $\delta_{z}^{(k)}$ :

$$
\begin{gathered}
v_{1}^{(k)}(t)=f_{k}(t), \quad v_{\overline{1}}^{(k)}(t)=\bar{R}_{k}(t) g_{k}(t)+\bar{G}_{k}(t) r_{k}(t), \\
v_{0}^{(k)}(t)=\frac{\int_{0}^{\infty} g_{k}(x+t) r_{k}(x) d x}{P\left(\beta_{k}>\tau_{k}\right)} .
\end{gathered}
$$

Then the mean residence time in the state $i \bar{d} \bar{x}$ is determined by the equality:

$$
\theta_{i \overline{d \bar{x}}}=\bigwedge_{k=1}^{N}\left[\delta_{d_{k}}^{(k)}-x_{k}\right]^{+}, x_{i}=0 .
$$

Define the transition probabilities of the embedded Markov chain (EMC) $\left\{\xi_{n} ; \mathrm{n} \geq 0\right\}$.

We introduce additional functions:

$$
\begin{aligned}
& w_{1, z}^{(k)}(t)=\left\{\begin{array}{l}
v_{1}^{(k)}(t), \mathrm{z}=\overline{1}, \\
0, \quad \mathrm{z}=0,1,
\end{array}\right.
\end{aligned}
$$

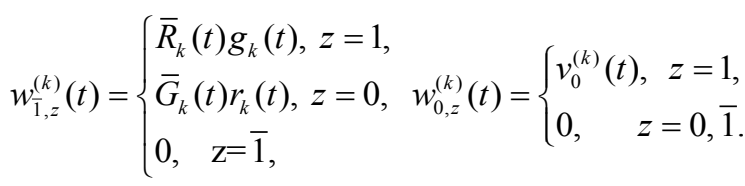

Note that the functions $f_{\overline{1}, 1}^{(k)}(t)=\frac{w_{\overline{1}, 1}^{(k)}(t)}{P\left(\tau_{k}>\beta_{k}\right)}$, $f_{\overline{1}, 0}^{(k)}(t)=\frac{w_{\overline{1}, 0}^{(k)}(t)}{P\left(\tau_{k}<\beta_{k}\right)}, k=\overline{1, N}$ are the densities of the distributions.

The probability densities of the transitions of the EMC $\left\{\xi_{n} ; \mathrm{n} \geq 0\right\}$ are as follows:

$$
p_{i \overline{d \bar{x}}}^{j \bar{d} \bar{y}}=\left\{\begin{array}{cc}
\frac{w_{d_{j}, d_{j}^{\prime}}^{(j)}\left(x_{j}+y_{i}\right) \prod_{\substack{k=1, k \neq j}}^{N} \bar{V}_{d_{k}}^{(k)}\left(x_{k}+y_{i}\right)}{\prod_{\substack{s=1, s \neq i}}^{N} \bar{V}_{d_{s}}^{(s)}\left(x_{s}\right)}, & j \neq i, y_{k}=x_{k}+y_{i}, \\
k \neq j, y_{j}=0, & \\
\frac{w_{d_{i}, d_{i}^{\prime}}^{(i)}\left(y_{k}-x_{k}\right) \prod_{\substack{k=1, k \neq i}}^{N} \bar{V}_{d_{k}}^{(k)}\left(y_{k}\right)}{\prod_{\substack{s=1, s \neq i}}^{N} \bar{V}_{d_{s}}^{(s)}\left(x_{s}\right)}, & k=i, y_{k}=x_{k}+t, \\
& k \neq i, y_{i}=x_{i}=0,
\end{array}\right.
$$

vectors $\bar{d}$ and $\bar{d}^{\prime}$ differ only in the $\mathrm{j}$-th component.

\section{Finding the stationary distribution of an embedded Markov chain}

To find the stationary characteristics of the reliability and efficiency of the system S, we find the stationary distribution of the EMC $\left\{\xi_{n} ; \mathrm{n} \geq 0\right\}$.

We introduce the following notation: let $\bar{x}=\left(x_{1}, x_{2}, \ldots, x_{N}\right), \quad$ then vectors $\quad(\bar{x}-t)^{(i)}$, $\left[\left(\bar{x}-x_{j}\right)^{(i)}, t\right]$, where $x_{j}=\bigwedge_{\substack{k=1, k \neq i}}^{N} x_{k}$, are defined as follows:

$(\bar{x}-t)_{k}^{(i)}=\left\{\begin{array}{l}x_{k}-t, k \neq i, \\ 0, \quad k=i,\end{array} \quad\left[\left(\bar{x}-x_{j}\right)^{(i)}, t\right]_{k}=\left\{\begin{array}{l}x_{k}-x_{j}, k \neq i, \\ t, \quad k=i .\end{array}\right.\right.$

Suppose that the stationary distribution of the EMC $\left\{\xi_{n} ; \mathrm{n} \geq 0\right\}$ has densities $\rho(i \bar{d} \bar{x})$, using (4), we compose a system of integral equations that they satisfy.

1. In the case of states $i \bar{d} \bar{x}, \bar{d}_{i}=\overline{1}$ next transitions are possible:
a) $\quad i \bar{d}^{(i)}(\bar{x}-t)^{(i)} \rightarrow i \bar{d} \bar{x}, 0<t<x_{j}, \bar{d}_{i}^{(i)}=1$,
b) $j \bar{d}^{(i)}\left[\left(\bar{x}-x_{j}\right)^{(i)}, t\right] \rightarrow i \bar{d} \bar{x}, t>0, \bar{d}_{i}^{(i)}=1$.

Consequently

$$
\begin{gathered}
\rho(i \bar{d} \bar{x})=\int_{0}^{x_{j}} \frac{v_{1}^{(i)}(t) \prod_{\substack{k=1, k \neq i}}^{N} \bar{V}_{d_{k}}^{(k)}\left(x_{k}\right)}{\prod_{\substack{s=1, s \neq i}}^{N} \bar{V}_{d_{s}}^{(s)}\left(x_{s}-t\right)} \rho\left(i \bar{d}^{(i)}(\bar{x}-t)^{(i)}\right) d t+ \\
+\int_{0}^{\infty} \frac{v_{1}^{(i)}\left(x_{j}+t\right) \prod_{\substack{k=1, k \neq i}}^{N} \bar{V}_{d_{k}}^{(k)}\left(x_{k}\right)}{\prod_{\substack{s=1, s \neq j}}^{N} \bar{V}_{d_{s}}^{(s)}\left(x_{s}-x_{j}\right)} \rho\left(j \bar{d}^{(i)}\left[\left(\bar{x}-x_{j}\right)^{(i)}, t\right]\right) d t .
\end{gathered}
$$

2. In the case of states $i \bar{d} \bar{x}, \bar{d}_{i}=0$, transitions will be:

a) $i \bar{d}^{(i)}(\bar{x}-t)^{(i)} \rightarrow i \bar{d} \bar{x}, 0<t<x_{j}, \bar{d}_{i}^{(i)}=\overline{1}$, 
b) $j \bar{d}^{(i)}\left[\left(\bar{x}-x_{j}\right)^{(i)}, t\right] \rightarrow i \bar{d} \bar{x}, t>0, \bar{d}_{i}^{(i)}=\overline{1}$.

Hence,

$$
\begin{gathered}
\rho(i \overline{d \bar{x}})=\int_{0}^{x_{j}} \frac{w_{\overline{1}, 0}^{(i)}(t) \prod_{\substack{k=1, k \neq i}}^{N} \bar{V}_{d_{k}}^{(k)}\left(x_{k}\right)}{\prod_{\substack{s=1, s \neq i}}^{N} \bar{V}_{d_{s}}^{(s)}\left(x_{s}-t\right)} \rho\left(i \bar{d}^{(i)}(\bar{x}-t)^{(i)}\right) d t+ \\
+\int_{0}^{\infty} \frac{w_{\overline{1}, 0}^{(i)}\left(x_{j}+t\right) \prod_{\substack{k=1, k \neq i}}^{N} \bar{V}_{d_{k}}^{(k)}\left(x_{k}\right)}{\prod_{\substack{s=1, s \neq j}}^{N} \bar{V}_{d_{s}}^{(s)}\left(x_{s}-x_{j}\right)} \rho\left(j \bar{d}^{(i)}\left[\left(\bar{x}-x_{j}\right)^{(i)}, t\right]\right) d t .
\end{gathered}
$$

3. For $i \bar{d} \bar{x}, \bar{d}_{i}=1$ transitions will be:
a) $i \bar{d}^{(i)}(\bar{x}-t)^{(i)} \rightarrow i \bar{d} \bar{x}, 0<t<x_{j}, \bar{d}_{i}^{(i)}=\overline{1}$,
b) $i \bar{d}^{(i)}(\bar{x}-t)^{(i)} \rightarrow i \bar{d} \bar{x}, 0<t<x_{j}, \bar{d}_{i}^{(i)}=0$,
c) $j \bar{d}^{(i)}\left[\left(\bar{x}-x_{j}\right)^{(i)}, t\right] \rightarrow i \bar{d} \bar{x}, t>0, \bar{d}_{i}^{(i)}=\overline{1}$,
d) $j \bar{d}^{(i)}\left[\left(\bar{x}-x_{j}\right)^{(i)}, t\right] \rightarrow i \bar{d} \bar{x}, t>0, \bar{d}_{i}^{(i)}=0$.

Therefore,

$$
\begin{gathered}
\rho(i \bar{d} \bar{x})=\int_{0}^{x_{j}} \frac{w_{\overline{1}, 1}^{(i)}(t) \prod_{\substack{k=1, k \neq i}}^{N} \bar{V}_{d_{k}}^{(k)}\left(x_{k}\right)}{\prod_{\substack{s=1, s \neq i}}^{N} \bar{V}_{d_{s}}^{(s)}\left(x_{s}-t\right)} \rho\left(i \bar{d}^{(i)}(\bar{x}-t)^{(i)}\right) d t+ \\
+\int_{0}^{x_{j}} \frac{w_{0,1}^{(i)}(t) \prod_{\substack{k=1, k \neq i}}^{N} \bar{V}_{d_{k}}^{(k)}\left(x_{k}\right)}{\prod_{\substack{s=1, s \neq i}}^{N} \bar{V}_{d_{s}}^{(s)}\left(x_{s}-t\right)} \rho\left(i \bar{d}^{(i)}(\bar{x}-t)^{(i)}\right) d t+
\end{gathered}
$$$$
+\int_{0}^{\infty} \frac{w_{\overline{1}, 1}^{(i)}\left(x_{j}+t\right) \prod_{\substack{k=1, k \neq i}}^{N} \bar{V}_{d_{k}}^{(k)}\left(x_{k}\right)}{\prod_{\substack{s=1, s \neq j}}^{N} \bar{V}_{d_{s}}^{(s)}\left(x_{s}-x_{j}\right)} \rho\left(j \bar{d}^{(i)}\left[\left(\bar{x}-x_{j}\right)^{(i)}, t\right]\right) d t+
$$$$
w_{0,1}^{(i)}\left(x_{j}+t\right) \prod^{N} \bar{V}_{d_{k}}^{(k)}\left(x_{k}\right)
$$$$
+\int_{0}^{\infty} \frac{\substack{k=1, k \neq i}}{\prod_{\substack{s=1, s \neq j}}^{N} \bar{V}_{d_{s}}^{(s)}\left(x_{s}-x_{j}\right)} \rho\left(j \bar{d}^{(i)}\left[\left(\bar{x}-x_{j}\right)^{(i)}, t\right]\right) d t .
$$

4. $\sum_{\bar{d} \in D} \sum_{i=1}^{N} \int_{R_{+}^{(N, i)}} \rho(i \bar{d} \bar{x}) d \bar{x}^{(i)}=1$

(normalization condition).
Denote by $\tilde{\rho}(i \overline{d \bar{x}})=\frac{\rho(i \overline{d \bar{x}})}{\prod_{k=1}^{N} \bar{V}_{d_{k}}^{(k)}\left(x_{k}\right)}$. Then the system of equations (5) - (8) will have the following form:

1. In the case of conditions $i \bar{d} \bar{x}, \bar{d}_{i}=\overline{1}$

$$
\begin{aligned}
& \tilde{\rho}(i \overline{d \bar{x}})=\int_{0}^{x_{j}} v_{1}^{(i)}(t) \tilde{\rho}\left(i \bar{d}^{(i)}(\bar{x}-t)^{(i)}\right) d t+ \\
& +\int_{0}^{\infty} v_{1}^{(i)}\left(x_{j}+t\right) \tilde{\rho}\left(j \bar{d}^{(i)}\left[\left(\bar{x}-x_{j}\right)^{(i)}, t\right]\right) d t .
\end{aligned}
$$

2. If $i \bar{d} \bar{x}, \bar{d}_{i}=0$, then

$$
\begin{aligned}
& \tilde{\rho}(i \bar{d} \bar{x})=\int_{0}^{x_{j}} w_{\overline{1}, 0}^{(i)}(t) \tilde{\rho}\left(i \bar{d}^{(i)}(\bar{x}-t)^{(i)}\right) d t+ \\
& +\int_{0}^{\infty} w_{\overline{1}, 0}^{(i)}\left(x_{j}+t\right) \tilde{\rho}\left(j \bar{d}^{(i)}\left[\left(\bar{x}-x_{j}\right)^{(i)}, t\right]\right) d t .
\end{aligned}
$$

3. In the case of states $i \overline{d x}, \bar{d}_{i}=1$

$$
\begin{gathered}
\tilde{\rho}(i \bar{d} \bar{x})=\int_{0}^{x_{j}} w_{\overline{1}, 1}^{(i)}(t) \tilde{\rho}\left(i \bar{d}^{(i)}(\bar{x}-t)^{(i)}\right) d t+ \\
+\int_{0}^{x_{j}} w_{0,1}^{(i)}(t) \tilde{\rho}\left(i \bar{d}^{(i)}(\bar{x}-t)^{(i)}\right) d t+ \\
+\int_{0}^{\infty} w_{\overline{1}, 1}^{(i)}\left(x_{j}+t\right) \tilde{\rho}\left(j \bar{d}^{(i)}\left[\left(\bar{x}-x_{j}\right)^{(i)}, t\right]\right) d t+ \\
+\int_{0}^{\infty} w_{0,1}^{(i)}\left(x_{j}+t\right) \tilde{\rho}\left(j \bar{d}^{(i)}\left[\left(\bar{x}-x_{j}\right)^{(i)}, t\right]\right) d t . \\
\sum_{\bar{d} \in D} \sum_{i=1}^{N} \int_{R_{+}^{(N, i)}} \tilde{\rho}(i \bar{d} \bar{x}) \prod_{k=1}^{N} \bar{V}_{d_{k}}^{(k)}\left(x_{k}\right) d \bar{x}^{(i)}=1
\end{gathered}
$$

(normalization condition).

Let's us introduce the following notation:

$$
\begin{gathered}
\rho_{z}^{(k)}=\left\{\begin{array}{l}
\rho^{(k)}, z=1, \overline{1}, \\
\bar{\rho}^{(k)}, z=0,
\end{array} \rho^{(k)}=\frac{1}{2+P\left(\beta_{k}>\tau_{k}\right)},\right. \\
\bar{\rho}^{(k)}=\frac{P\left(\beta_{k}>\tau_{k}\right)}{2+P\left(\beta_{k}>\tau_{k}\right)}, \quad \bar{\rho}^{(k)}=\rho^{(k)} \cdot P\left(\beta_{k}>\tau_{k}\right) .
\end{gathered}
$$

Lemma. The solution to the system of equations (9) - (12) has the following form:

$$
\tilde{\rho}(i \overline{d \bar{x}})=\rho_{0} \prod_{k=1}^{N} \rho_{d_{k}}^{(k)},
$$

where the constant $\rho_{0}$ is found from the normalization condition (12).

Proof. By substitution, we verify that the functions $\tilde{\rho}(i \overline{d \bar{x}})=\rho_{0} \prod_{k=1}^{N} \rho_{d_{k}}^{(k)}$ satisfy the system of equations (9) -

(12) for any value $\rho_{0}$. 
1. In the case $i \bar{d} \bar{x}, \bar{d}_{i}=\overline{1}$

$$
\begin{aligned}
\tilde{\rho}(i \overline{d \bar{x}}) & =\rho_{0} \prod_{k=1}^{N} \rho_{d_{k}}^{(k)}=\int_{0}^{x_{j}} v_{1}^{(i)}(t) \rho_{0} \prod_{k=1}^{N} \rho_{d_{k}}^{(k)} d t+\int_{0}^{\infty} v_{1}^{(i)}\left(x_{j}+t\right) \rho_{0} \prod_{k=1}^{N} \rho_{d_{k}}^{(k)} d t=\rho_{0} \prod_{k=1}^{N} \rho_{d_{k}}^{(k)}\left(\int_{0}^{x_{j}} v_{1}^{(i)}(t) d t+\int_{0}^{\infty} v_{1}^{(i)}\left(x_{j}+t\right) d t\right)= \\
& =\rho_{0} \prod_{k=1}^{N} \rho_{d_{k}}^{(k)}\left(\int_{0}^{x_{j}} v_{1}^{(i)}(t) d t+\int_{x_{j}}^{\infty} v_{1}^{(i)}\left(t^{\prime}\right) d t^{\prime}\right)=\rho_{0} \prod_{k=1}^{N} \rho_{d_{k}}^{(k)} \int_{0}^{\infty} v_{1}^{(i)}(t) d t=\rho_{0} \prod_{k=1}^{N} \rho_{d_{k}}^{(k)} \int_{0}^{\infty} f_{i}(t) d t=\rho_{0} \prod_{k=1}^{N} \rho_{d_{k}}^{(k)} .
\end{aligned}
$$

2. If $i \bar{d} \bar{x}, \bar{d}_{i}=0$ then

$$
\begin{aligned}
\tilde{\rho}(i \overline{d \bar{x}}) & =\rho_{0} \prod_{k=1}^{N} \rho_{d_{k}}^{(k)}=\int_{0}^{x_{j}} w_{\overline{1}, 0}^{(i)}(t) \rho_{0} \prod_{k=1}^{N} \rho_{d_{k}}^{(k)} d t+\int_{0}^{\infty} w_{\overline{1}, 0}^{(i)}\left(x_{j}+t\right) \rho_{0} \prod_{k=1}^{N} \rho_{d_{k}}^{(k)} d t=\rho_{0} \prod_{k=1}^{N} \rho_{d_{k}}^{(k)}\left(\int_{0}^{x_{j}} w_{\overline{1}, 0}^{(i)}(t) d t+\int_{0}^{\infty} w_{\overline{1}, 0}^{(i)}\left(x_{j}+t\right) d t\right)= \\
& =\rho_{0} \prod_{k=1}^{N} \rho_{d_{k}}^{(k)}\left(\int_{0}^{x_{j}} w_{\overline{1}, 0}^{(i)}(t) d t+\int_{x_{j}}^{\infty} w_{\overline{1}, 0}^{(i)}\left(t^{\prime}\right) d t^{\prime}\right)=\rho_{0} \prod_{k=1}^{N} \rho_{d_{k}}^{(k)} \int_{0}^{\infty} w_{\overline{1}, 0}^{(i)}(t) d t=\rho_{0} \prod_{k=1}^{N} \rho_{d_{k}}^{(k)} \int_{0}^{\infty} f_{\overline{1}, 0}^{(i)}(t) d t=\rho_{0} \prod_{k=1}^{N} \rho_{d_{k}}^{(k)} .
\end{aligned}
$$

3. For $i \bar{d} \bar{x}, \bar{d}_{i}=1$

$$
\begin{aligned}
& \tilde{\rho}(i \overline{d \bar{x}})=\rho_{0} \prod_{k=1}^{N} \rho_{d_{k}}^{(k)}=\int_{0}^{x_{j}} w_{\overline{1}, 1}^{(i)}(t) \rho_{0} \prod_{k=1}^{N} \rho_{d_{k}}^{(k)} d t+\int_{0}^{x_{j}} w_{0,1}^{(i)}(t) \rho_{0} \prod_{k=1}^{N} \rho_{d_{k}}^{(k)} d t+\int_{0}^{\infty} w_{\overline{1}, 1}^{(i)}\left(x_{j}+t\right) \rho_{0} \prod_{k=1}^{N} \rho_{d_{k}}^{(k)} d t+ \\
& +\int_{0}^{\infty} w_{0,1}^{(i)}\left(x_{j}+t\right) \rho_{0} \prod_{k=1}^{N} \rho_{d_{k}}^{(k)} d t=\rho_{0} \prod_{k=1}^{N} \rho_{d_{k}}^{(k)}\left(\int_{0}^{x_{j}} w_{\overline{1}, 1}^{(i)}(t) d t+\int_{0}^{x_{j}} w_{0,1}^{(i)}(t) d t+\int_{0}^{\infty} w_{\overline{1}, 1}^{(i)}\left(x_{j}+t\right) d t+\int_{0}^{\infty} w_{0,1}^{(i)}\left(x_{j}+t\right) d t\right)= \\
& =\rho_{0} \prod_{k=1}^{N} \rho_{d_{k}}^{(k)}\left(\int_{0}^{x_{j}} w_{\overline{1}, 1}^{(i)}(t) d t+\int_{x_{j}}^{\infty} w_{\overline{1}, 1}^{(i)}\left(t^{\prime}\right) d t^{\prime}+\int_{0}^{x_{j}} w_{0,1}^{(i)}(t) d t+\int_{x_{j}}^{\infty} w_{0,1}^{(i)}\left(t^{\prime}\right) d t^{\prime}\right)==\rho_{0} \prod_{k=1}^{N} \rho_{d_{k}}^{(k)}\left(\int_{0}^{\infty} w_{\overline{1}, 1}^{(i)}(t) d t+\int_{0}^{\infty} w_{0,1}^{(i)}(t) d t\right)= \\
& =\rho_{0} \prod_{k=1}^{N} \rho_{d_{k}}^{(k)}\left(\int_{0}^{\infty} \bar{R}_{i}(t) g_{i}(t) d t+\int_{0}^{\infty} v_{0}^{(i)}(t) d t\right)=\rho_{0} \prod_{k=1}^{N} \rho_{d_{k}}^{(k)}\left(P\left(\tau_{i}>\beta_{i}\right)+\int_{0}^{\infty} d t \int_{0}^{\infty} g_{i}(y+t) r_{i}(y) d y\right)= \\
& =\rho_{0} \prod_{k=1}^{N} \rho_{d_{k}}^{(k)}\left(P\left(\tau_{i}>\beta_{i}\right)+\int_{0}^{\infty} r_{i}(t) \bar{G}_{i}(t) d t\right)=\rho_{0} \prod_{k=1}^{N} \rho_{d_{k}}^{(k)}\left(P\left(\tau_{i}>\beta_{i}\right)+P\left(\tau_{i} \leq \beta_{i}\right)\right)=\rho_{0} \prod_{k=1}^{N} \rho_{d_{k}}^{(k)} .
\end{aligned}
$$

The lemma is proved.

Consequently, the stationary distribution of EMC $\left\{\xi_{n} ; \mathrm{n} \geq 0\right\}$ of the system in question has the following form:

$$
\rho(i \bar{d} \bar{x})=\rho_{0} \prod_{k=1}^{N} \rho_{d_{k}}^{(k)} \bar{V}_{d_{k}}^{(k)}\left(x_{k}\right),
$$

where the constant $\rho_{0}$ is found from the normalization condition.

\section{Finding the stationary characteristics of the system}

To find the stationary availability factor $K_{a}$, mean stationary operating time of the system to failure $T_{+}$, mean stationary restoration time $T_{-}$, we use the following formulas presented in $[8,12]$ :

$$
K_{a}=\frac{\int_{E_{+}} m(e) \rho(d e)}{\int_{E} m(e) \rho(d e)}=\frac{T_{+}}{T_{+}+T_{-}},
$$

$$
T_{+}=\frac{\int_{E_{+}} m(e) \rho(d e)}{\int_{E_{+}} P\left(e, E_{-}\right) \rho(d e)}, \quad T_{-}=\frac{\int_{E_{-}} m(e) \rho(d e)}{\int_{E_{+}} P\left(e, E_{-}\right) \rho(d e)}
$$

where $\rho(d e)$ is stationary distribution of EMC $\left\{\xi_{n} ; n \geq 0\right\}, P\left(e, E_{-}\right)$- transition probabilities of the EMC $\left\{\xi_{n} ; n \geq 0\right\}$ to a subset of fault states $E_{-}, m(e)$ is mean residence time of the semi-Markov process in the state $e \in E$.

Consider the concept of system failure: based on an analysis of the structure of the system, on the set of all the vectors $D=\{\bar{d}\}$ a structural function [11] $g(\bar{d})$ is defined as:

$$
g(\bar{d})=\left\{\begin{array}{l}
1, \text { if the system is operational for } \\
\text { a given combination of } \bar{d} \text { components; } \\
0, \text { if the system is in failure. }
\end{array}\right.
$$


The set of vector $\bar{d}$ values at which the system is operational, we denote $D_{+}$, and the set of values at which the system is in failure, by $D_{-}$, i.e. $D_{+}=\{\bar{d}: g(\bar{d})=1\}, \quad D_{-}=\{\bar{d}: g(\bar{d})=0\} . \quad$ By assumption, $D=D_{+} \cup D_{-}, D_{+} \cap D_{-}=\varnothing$.

In accordance with the choice of $D_{+}$and $D_{-}$, the phase state space $E$ is divided into sets $E_{+}$and $E_{-}$of operable and fault states. $E=E_{+} \cup E_{-}, E_{+} \cap E_{-}=\varnothing$.

We introduce the following notations for finding $T_{+}$, $T_{-}$:

$D_{+}^{0}$ is a set of vectors $\bar{d} \in D_{+}$such that a change in the state of one component puts the system in a failure state (in $D_{-}$),
$G(\bar{d})$ is the set of numbers of the components of the vector $\bar{d} \in D_{+}^{0}$, changing the value of each of which translates the vector $\bar{d}$ into $D_{-}$.

We find the numerator of the first of formulas (15). Using (2), we determine the mean residence times in the states:

$$
E \theta_{i \overline{i \bar{x}}}=\frac{\int_{0}^{\infty} \prod_{k=1}^{N} \bar{V}_{d_{k}}^{(k)}\left(x_{k}+t\right) d t}{\prod_{\substack{s=1 \\ s \neq i}}^{N} \bar{V}_{d_{s}}^{(s)}\left(x_{s}\right)}, x_{i}=0
$$

Using (16) and stationary distribution (14), we obtain:

$$
\begin{aligned}
& \int_{E_{+}} m(e) \rho(d e)=\sum_{\bar{d} \in D_{+}} \sum_{i=1}^{N} \int_{0}^{\infty} \ldots \int_{0}^{\infty} \rho_{0} \prod_{k=1}^{N} \rho_{d_{k}}^{(k)} \prod_{\substack{j=1,1 \\
j \neq i}}^{N} \bar{V}_{d_{j}}^{(j)}\left(x_{j}\right) d \bar{x}^{(i)} \int_{0}^{\infty} \frac{\prod_{k=1}^{N} \bar{V}_{d_{k}}^{(k)}\left(x_{k}+t\right)}{\prod_{\substack{s=1, s \neq i}}^{N} \bar{V}_{d_{s}}^{(s)}\left(x_{s}\right)} d t=\sum_{\bar{d} \in D_{+}} \rho_{0} \prod_{k=1}^{N} \rho_{d_{k}}^{(k)} \sum_{i=1}^{N} \int_{0}^{\infty} \ldots \int_{0}^{\infty} d \bar{x}^{(i)} \int_{0}^{\infty} \prod_{k=1}^{N} \bar{V}_{d_{k}}^{(k)}\left(x_{k}+t\right) d t= \\
& =\sum_{\bar{d} \in D_{+}} \rho_{0} \prod_{k=1}^{N} \rho_{d_{k}}^{(k)} \prod_{k=1}^{N} \int_{0}^{\infty} \bar{V}_{d_{k}}^{(k)}\left(x_{k}\right) d x_{k}=\rho_{0} \sum_{\bar{d} \in D_{+}} \prod_{k=1}^{N} \rho_{d_{k}}^{(k)} E \delta_{d_{k}}^{(k)}=\rho_{0} \prod_{k=1}^{N} \rho^{(k)} \cdot \sum_{\bar{d} \in D_{+}} \prod_{k: d_{k}=1} E \alpha_{k} \cdot \prod_{k: d_{k}=\overline{1}} E\left(\beta_{k} \wedge \tau_{k}\right) \cdot \prod_{k: d_{k}=0} P\left(\beta_{k}>\tau_{k}\right) E\left(\left[\beta_{k}-\tau_{k}\right]^{+}\right), \\
& \int_{E_{-}} m(e) \rho(d e)=\rho_{0} \sum_{\bar{d} \in D_{-}} \prod_{k=1}^{N} \rho_{d_{k}}^{(k)} E \delta_{d_{k}}^{(k)}=\rho_{0} \prod_{k=1}^{N} \rho^{(k)} \cdot \sum_{\bar{d} \in D_{-}} \prod_{k: d_{k}=1} E \alpha_{k} \cdot \prod_{k: d_{k}=\overline{1}} E\left(\beta_{k} \wedge \tau_{k}\right) \cdot \prod_{k: d_{k}=0} P\left(\beta_{k}>\tau_{k}\right) E\left(\left[\beta_{k}-\tau_{k}\right]^{+}\right) . \\
& \int_{E_{+}} P\left(e, E_{-}\right) \rho(d e)=\rho_{0} \prod_{k=1}^{N} \rho^{(k)} \cdot \sum_{\bar{d} \in D_{+}^{0}} \sum_{j \in G(\bar{d})} I\left(d_{j}\right) \cdot \prod_{\substack{k: d_{k}=1, k \neq j}} E \alpha_{k} \cdot \prod_{\substack{k: d_{k}=\overline{1}, k \neq j}} E\left(\beta_{k} \wedge \tau_{k}\right) \cdot \prod_{\substack{k, d_{k}=0, k \neq j}} P\left(\beta_{k}>\tau_{k}\right) E\left(\left[\beta_{k}-\tau_{k}\right]^{+}\right),
\end{aligned}
$$

where $I\left(d_{j}\right)=\left\{\begin{array}{c}P\left(\beta_{j}>\tau_{j}\right), \text { if } d_{j}=\overline{1}, \\ 1, \quad \text { if } d_{j}=1,0 .\end{array}\right.$

$$
\begin{gathered}
K_{a}=\frac{\sum_{d \in D_{+}} \prod_{k=1}^{N} \rho_{d_{k}}^{(k)} \prod_{k=1}^{N} E \delta_{d_{k}}^{(k)}}{\sum_{\bar{d} \in D}^{N} \prod_{k=1}^{N} \rho_{d_{k}}^{(k)} \prod_{k=1}^{N} E \delta_{d_{k}}^{(k)}}=\frac{\sum_{\bar{d} \in D_{+}} \prod_{k=1}^{N} \rho_{d_{k}}^{(k)} E \delta_{d_{k}}^{(k)}}{\prod_{k=1}^{N} \rho_{d_{k}}^{(k)}\left(E \delta_{1}^{(k)}+E \delta_{\overline{1}}^{(k)}+P\left(\beta_{k}>\tau_{k}\right) E \delta_{0}^{(k)}\right)}=\frac{\sum_{d \in D_{+}} \prod_{k=1}^{N} \rho_{d_{k}}^{(k)} E \delta_{d_{k}}^{(k)}}{\prod_{k=1}^{N} \rho_{d_{k}}^{(k)}\left(E \alpha_{k}+E\left(\beta_{k} \wedge \tau_{k}\right)+P\left(\beta_{k}>\tau_{k}\right) E\left[\beta_{k}-\tau_{k}\right]^{+}\right)}= \\
=\frac{\sum_{\bar{d} \in D_{+}} \prod_{k: d_{k}=1} E \alpha_{k} \cdot \prod_{k: d_{k}=1} E\left(\beta_{k} \wedge \tau_{k}\right) \cdot \prod_{k: d_{k}=0} P\left(\beta_{k}>\tau_{k}\right) E\left(\left[\beta_{k}-\tau_{k}\right]^{+}\right)}{\prod_{k=1}^{N}\left(E \alpha_{k}+E \beta_{k}\right)},
\end{gathered}
$$

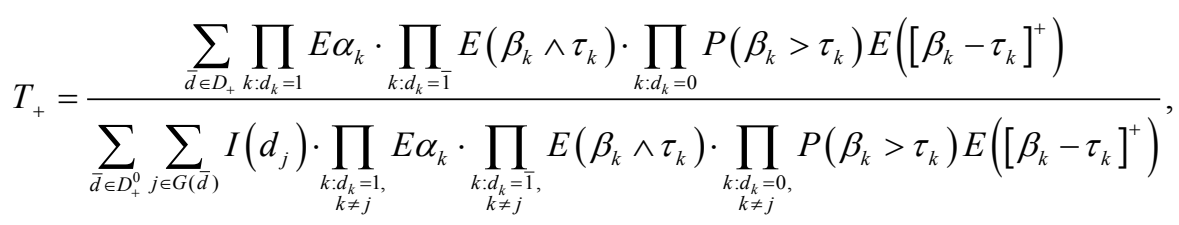$$
T_{-}=\frac{\sum_{\bar{d} \in D_{-} k: d_{k}=1} E \alpha_{k} \cdot \prod_{k: d_{k}=\overline{1}} E\left(\beta_{k} \wedge \tau_{k}\right) \cdot \prod_{k: d_{k}=0} P\left(\beta_{k}>\tau_{k}\right) E\left(\left[\beta_{k}-\tau_{k}\right]^{+}\right)}{\sum_{\bar{d} \in D_{+}^{0}} \sum_{j \in G(\bar{d})} I\left(d_{j}\right) \cdot \prod_{\substack{k: d_{k}=1, k \neq j}} E \alpha_{k} \cdot \prod_{\substack{k: d_{k}=\overline{1} \\ k \neq j}} E\left(\beta_{k} \wedge \tau_{k}\right) \cdot \prod_{\substack{k: d_{k}=0, k \neq j}} P\left(\beta_{k}>\tau_{k}\right) E\left(\left[\beta_{k}-\tau_{k}\right]^{+}\right)},
$$ 
where

$$
E\left(\beta_{k} \wedge \tau_{k}\right)=\int_{0}^{\infty} \bar{G}_{k}(t) \bar{R}_{k}(t) d t, E\left(\left[\beta_{k}-\tau_{k}\right]^{+}\right)=\frac{\int_{0}^{\infty} \bar{G}_{k}(t) R_{k}(t) d t}{P\left(\beta_{k}>\tau_{k}\right)} .
$$

In the transformations, we use the following formulas $[8,12]$ :

$$
\begin{gathered}
\sum_{j=1}^{N} \int_{0}^{\infty} \ldots \int_{0}^{\infty} \bar{F}_{j}(t)\left(\prod_{\substack{k=1, k \neq j}}^{N} \bar{F}_{k}\left(y_{k}+t\right) d y_{k}\right) d t=\prod_{j=1}^{N} E \alpha_{j}, \\
\sum_{k=1}^{N} \int_{R_{+}^{N, k}} \int_{0}^{\infty} f_{i}\left(x_{i}+t\right) \prod_{\substack{r=1, r \neq i}}^{N} \bar{F}_{r}\left(x_{r}+t\right) d \bar{x}^{(k)} d t=\prod_{\substack{k=1, k \neq i}}^{N} E \alpha_{k},
\end{gathered}
$$

where $F_{k}(t)$ are distribution functions of independent non négative random variables $\alpha_{k}$ with mathematical expectations $E \alpha_{k}$.

We find the efficiency characteristics of this system, such as the mean specific income per calendar time unit $(\mathrm{S})$ and the mean specific expenses per time unit of upstate $(C)$.To determine them, we use the formulas [10]:

$$
S=\frac{\int_{E} m(e) f_{s}(e) \rho(d e)}{\int_{E} m(e) \rho(d e)}, \quad C=\frac{\int_{E} m(e) f_{c}(e) \rho(d e)}{\int_{E_{+}} m(e) \rho(d e)},
$$

where $f_{s}(e), f_{c}(e)$ are functions denoting income and expenses in each state.

$$
\text { Let } f_{s}(e)=\left\{\begin{array}{l}
c_{1}, e \in E_{+}, \\
-c_{2}, e \in E_{-},
\end{array} \quad f_{c}(e)=\left\{\begin{array}{l}
0, e \in E_{+}, \\
c_{2}, e \in E_{-} .
\end{array}\right.\right.
$$

where $c_{1}$ is the income per time unit of the system upstate, $c_{2}$ denotes expenses per time system failure.

Then

$$
\begin{gathered}
S=\frac{c_{1} \sum_{\bar{d} \in D_{+}} \prod_{k: d_{k}=1} E \alpha_{k} \cdot \prod_{k: d_{k}=\overline{1}} E\left(\beta_{k} \wedge \tau_{k}\right) \cdot \prod_{k: d_{k}=0} P\left(\beta_{k}>\tau_{k}\right) E\left(\left[\beta_{k}-\tau_{k}\right]^{+}\right)}{\prod_{k=1}^{N}\left(E \alpha_{k}+E \beta_{k}\right)} \\
-\frac{-c_{2} \sum_{\bar{d} \in D_{-}: \prod_{k}=1} E \alpha_{k} \cdot \prod_{k: d_{k}=\overline{1}} E\left(\beta_{k} \wedge \tau_{k}\right) \cdot \prod_{k: d_{k}=0} P\left(\beta_{k}>\tau_{k}\right) E\left(\left[\beta_{k}-\tau_{k}\right]^{+}\right)}{\prod_{k=1}^{N}\left(E \alpha_{k}+E \beta_{k}\right)}
\end{gathered}
$$

$$
C=\frac{c_{2} \sum_{\bar{d} \in D_{-}} \prod_{k: d_{k}=1} E \alpha_{k} \cdot \prod_{k: d_{k}=\overline{1}} E\left(\beta_{k} \wedge \tau_{k}\right) \cdot \prod_{k: d_{k}=0} P\left(\beta_{k}>\tau_{k}\right) E\left(\left[\beta_{k}-\tau_{k}\right]^{+}\right)}{\sum_{\bar{d} \in D_{+}} \prod_{k: d_{k}=1} E \alpha_{k} \cdot \prod_{k: d_{k}=\overline{1}} E\left(\beta_{k} \wedge \tau_{k}\right) \cdot \prod_{k: d_{k}=0} P\left(\beta_{k}>\tau_{k}\right) E\left(\left[\beta_{k}-\tau_{k}\right]^{+}\right)} .
$$

Consider special cases of serial and parallel connection of system components.

1. Parallel connection.
In this case, a system failure occurs only if all components of the system are in failure. Formulas (17) (19) take the form:

$$
\begin{aligned}
K_{a}= & \frac{\sum_{i=1}^{N} P\left(\beta_{i}>\tau_{i}\right) E\left(\left[\beta_{i}-\tau_{i}\right]^{+}\right) \prod_{\substack{k=1, k \neq i}}^{N}\left(E \alpha_{k}+E\left(\beta_{k} \wedge \tau_{k}\right)\right)}{\prod_{k=1}^{N}\left(E \alpha_{k}+E \beta_{k}\right)}+ \\
+ & \frac{\prod_{i=1}^{N}\left(E \alpha_{i}+E\left(\beta_{i} \wedge \tau_{i}\right)\right)}{\prod_{k=1}^{N}\left(E \alpha_{k}+E \beta_{k}\right)}
\end{aligned}
$$

$$
T_{-}=\frac{\prod_{i=1}^{N} P\left(\beta_{i}>\tau_{i}\right) E\left(\left[\beta_{i}-\tau_{i}\right]^{+}\right)}{\sum_{i=1}^{N}\left(P\left(\beta_{i}>\tau_{i}\right) \prod_{\substack{k=1, k \neq i}}^{N} P\left(\beta_{k}>\tau_{k}\right) E\left(\left[\beta_{k}-\tau_{k}\right]^{+}\right)\right)},
$$

$$
\begin{gathered}
T_{+}=\frac{\sum_{i=1}^{N} P\left(\beta_{i}>\tau_{i}\right) E\left(\left[\beta_{i}-\tau_{i}\right]^{+}\right) \prod_{\substack{k=1, k \neq i}}^{N}\left(E \alpha_{k}+E\left(\beta_{k} \wedge \tau_{k}\right)\right)}{\sum_{i=1}^{N}\left(P\left(\beta_{i}>\tau_{i}\right) \prod_{\substack{k=1, k \neq i}}^{N} P\left(\beta_{k}>\tau_{k}\right) E\left(\left[\beta_{k}-\tau_{k}\right]^{+}\right)\right)}+ \\
+\frac{\prod_{i=1}^{N}\left(E \alpha_{i}+E\left(\beta_{i} \wedge \tau_{i}\right)\right)}{\sum_{i=1}^{N}\left(P\left(\beta_{i}>\tau_{i}\right) \prod_{\substack{k=1, k \neq i}}^{N} P\left(\beta_{k}>\tau_{k}\right) E\left(\left[\beta_{k}-\tau_{k}\right]^{+}\right)\right)} .
\end{gathered}
$$

2. Serial connection.

In this case, a system failure occurs if at least one of the components is in a failure. Formulas (17) - (19) take the form:

$$
\begin{gathered}
K_{a}=\frac{\prod_{i=1}^{N}\left(E \alpha_{i}+E\left(\beta_{i} \wedge \tau_{i}\right)\right)}{\prod_{k=1}^{N}\left(E \alpha_{k}+E \beta_{k}\right)}, \\
T_{+}=\frac{\prod_{i=1}^{N}\left(E \alpha_{i}+E\left(\beta_{i} \wedge \tau_{i}\right)\right)}{\sum_{i=1}^{N}\left(P\left(\beta_{i}>\tau_{i}\right) \prod_{\substack{k=1, k \neq i}}^{N}\left(E \alpha_{k}+E\left(\beta_{k} \wedge \tau_{k}\right)\right)\right)}, \\
\left.+\frac{\sum_{i=1}^{N} P\left(\beta_{i}>\tau_{i}\right) E\left(\left[\beta_{i}-\tau_{i}\right]^{+}\right) \prod_{\substack{k=1, k \neq i}}^{N}\left(E \alpha_{k}+E\left(\beta_{k} \wedge \tau_{k}\right)\right)}{\sum_{i=1}^{N}\left(P\left(\beta_{i}>\tau_{i}\right) \prod_{\substack{k=1, k \neq i}}^{N}\left(E \alpha_{k}+E\left(\beta_{k} \wedge \tau_{k}\right)\right)\right.}\right) \\
\prod_{i=1}^{N} P\left(\beta_{i}>\tau_{i}\right) E\left(\left[\beta_{i}-\tau_{i}\right]^{+}\right) \\
\left.P\left(\beta_{i}\right) \prod_{\substack{k=1, k \neq i}}^{N}\left(E \alpha_{k}+E\left(\beta_{k} \wedge \tau_{k}\right)\right)\right)
\end{gathered}
$$


In the case $\tau_{k}=h_{k}=$ const,

$$
\begin{gathered}
E\left(\beta_{k} \wedge \tau_{k}\right)=E\left(\beta_{k} \wedge h_{k}\right)=\int_{0}^{h_{k}} \bar{G}_{k}(t) d t \\
E\left(\left[\beta_{k}-\tau_{k}\right]^{+}\right)=E\left(\left[\beta_{k}-h_{k}\right]^{+}\right)=\int_{h_{k}}^{\infty} \bar{G}_{k}(t) d t / P\left(\beta_{k}>h_{k}\right) .
\end{gathered}
$$

As an illustrative example of the use of formulas (17) - (21), consider a system consisting of 3 components with parallel connection in which operating time of components $K_{1}, \quad K_{2}, \quad K_{3} \quad$ are $E \alpha_{1}=8 \mathrm{~h}$, $E \alpha_{2}=E \alpha_{3}=6 \mathrm{~h}$; recovery times of $K_{1}, K_{2}, K_{3}$ are $E \beta_{1}=0.71 \mathrm{~h}, E \beta_{2}=E \beta_{3}=0.83 \mathrm{~h}$, RVs $\alpha_{i}, \beta_{i}$ have 5th order Erlang distribution; $c_{1}=10$ c.u., $c_{2}=15$ c.u. Each component has a non-random time reserve ( $\left.R_{i}(t)=1\left(t-h_{i}\right), h_{1}=h_{2}=h_{3}=h\right)$, which varies from 0 to 0.7 hours in 0.1 increments. The corresponding values of $\quad T_{+}\left(h_{1}, h_{2}, h_{3}\right), \quad T_{-}\left(h_{1}, h_{2}, h_{3}\right), \quad K_{a}\left(h_{1}, h_{2}, h_{3}\right)$, $C\left(h_{1}, h_{2}, h_{3}\right), S\left(h_{1}, h_{2}, h_{3}\right)$ of the system for the specified distribution were calculated. The results are presented in Table 1.

Table 1. The influence of the time reserve value on the system reliability and efficiency characteristics.

\begin{tabular}{|c|c|c|c|c|c|}
\hline $\begin{array}{c}h, \\
\mathbf{h}\end{array}$ & $\begin{array}{c}T_{-}, \\
\mathbf{h}\end{array}$ & $\begin{array}{c}T_{+}, \\
\mathbf{h}\end{array}$ & $K_{a}$ & $\begin{array}{c}\mathbf{C}, \\
\mathbf{c . u .} / \mathbf{h} \\
* 10^{-3}\end{array}$ & $\begin{array}{c}\mathbf{S}, \\
\mathbf{c . u .} / \mathbf{h}\end{array}$ \\
\hline 0 & 0.2632 & 316.51 & 0.99917 & 12.47 & 9.9792 \\
\hline 0.1 & 0.2297 & 388.01 & 0.99941 & 8.882 & 9.9852 \\
\hline 0.2 & 0.1982 & 499.30 & 0.9996 & 5.953 & 9.9901 \\
\hline 0.3 & 0.1712 & 693.22 & 0.99975 & 3.705 & 9.9938 \\
\hline 0.4 & 0.1499 & $1.06 * 10^{3}$ & 0.99986 & 2.122 & 9.9965 \\
\hline 0.5 & 0.1336 & $1.798 * 10^{3}$ & 0.99993 & 1.114 & 9.9981 \\
\hline 0.6 & 0.1211 & $3.384 * 10^{3}$ & 0.99996 & 0.537 & 9.9991 \\
\hline 0.7 & 0.1115 & $7.023 * 10^{3}$ & 0.99998 & 0.238 & 9.9996 \\
\hline
\end{tabular}

Analysis of the data in the table shows the significant effect of the time reserve on reliability and efficiency characteristics.

These formulas can be used to analyse the reliability of multi-component systems "gas pipeline - underground gas storage - consumer"; oil pipeline with tank farms (for storing intermediate oil reserves); electric power systems, where the time reserve for the component is ensured by the presence of an energy storage device.

\section{Conclusion}

In this paper, a semi-Markov model of a multicomponent system with a component-wise random instantly replenished time reserve is constructed. The analysis of the influence of the amount of time reserve on the obtained reliability and efficiency characteristics is carried out.

In the future, it is planned to build hidden semiMarkov models of multi-component systems with an element-wise time reserve for analysing the functioning of energy systems.

The results of this work can be used to build semiMarkov models of systems with various types and strategies for using the time reserve, engineering calculations and solving optimization problems associated with the use of the time reserve.

The research was carried out within the state assignment of the Minobrnauki of Russia (No. 1.10513.2018/11.12), with financial support by RFBR (project No. 19-01-00704a).

\section{References}

1. G.N. Cherkesov, Reliability of Technical Systems with Time Redundancy (Sovietskoe Radio, Moscow, 1974)

2. B.P. Kredentser, Prediction of Reliability of Systems with Time Redundancy (Naukova Dumka, Kiev, 1978)

3. V.Y. Kopp, Yu.E. Obzherin, A.I. Peschanskiy, Stochastic models of automized system with time reservation (Publishing of Sevastopol State Technical University, Sevastopol, 2000)

4. I.A. Ushakov, Probabilistic Reliability Models (Wiley, San Diego, Calif., 2012)

5. Yu. N. Rudenko, I.A. Ushakov, Reliability of Energy Systems, 2nd ed. (Nauka, Novosibirsk, 1989)

6. Yu.E. Obzherin, S.M. Sidorov, M.M. Nikitin, Analysis of reliability of systems with componentwise storages, E3S Web of Conférences 58, 02024. (2018).

7. N. Limnios, G. Oprisan, Semi-Markov Processes and Reliability (Springer Science+Business Media, New York, 2001)

8. V.S. Korolyuk, A.F. Turbin, Markovian restoration processes in the problems of system reliability (Naukova Dumka, Kiev, 1982)

9. V.S. Korolyuk, V.V. Korolyuk, Stochastic Models of Systems (Springer Science+Business Media, Dordrecht, 1999)

10. V.M. Shurenkov, Ergodic Markovian processes (Nauka, Moscow, 1989)

11. F. Beichelt, P. Tittmann, Reliability and Maintenance-Networks and Systems. Mathematical Models (Chapman \& Hall, CRC Press, Boca Raton, London, New York, 2012)

12. A.N. Korlat, V.N. Kuznetsov, M.M. Novikov, A.F. Turbin, Semi-Markov Models of Recoverable Systems and Queuing Systems (Stiinta, Chisinau, 1991) 